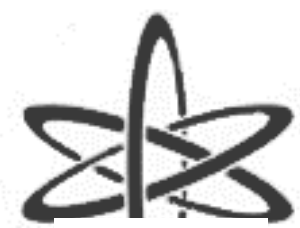

BJRS
BRAZILIAN JOURNAL

$\mathrm{OF}$

RADIATION SCIENCES

05-01 (2017) 01-12

\title{
Aspectos metrológicos na estimativa da atividade administrada em pacientes de medicina nuclear
}

\author{
A. Ruzzarin ${ }^{\mathrm{a}}$; A. M. Xavier; A. Iwahara ${ }^{\mathrm{a}}$; L. Tahuata ${ }^{\mathrm{a}}$ \\ ${ }^{a}$ Instituto de Radioproteção e Dosimetria/LNMRI, Rio de Janeiro, RJ, Brasil \\ ${ }^{b}$ Comissão Nacional de Energia Nuclear - Escritório de Porto Alegre/SEMRI, Rio de Janeiro, RJ, Brasil \\ anelise@bolsista.ird.gov.br
}

\section{RESUMO}

A fim de investigar a qualidade de desempenho das medições de rotina dos Serviços de Medicina Nuclear (SMN), o Laboratório Nacional de Metrologia de Radiação Ionizante / Instituto de Radioproteção e Dosimetria (LNMRI / IRD), vem conduzindo desde 1998 um programa de comparação de medições de Atividade de radiofármacos administradas a pacientes em medicina nuclear. Os fatores de correção na atividade administrada são determinados a partir do resultado da análise do desempenho dos ativímetros, a fim de determinar com maior precisão a atividade a ser administrada aos pacientes.

Palavras chave: medicina nuclear, ativímetro, atividade.

\begin{abstract}
In order to investigate the performance quality of routine measurements of Nuclear Medicine Services (NMS), the National Metrology Laboratory of Ionizing Radiation/Institute of Radiation Protection and Dosimetry (LNMRI/IRD) has been conducting, since 1998, a program of comparison for activity measurements of radiopharmaceuticals administered to patients in nuclear medicine. Correction factors are determined from the result of performance analysis in order to determine with better accuracy the activity to be administered to the patients.
\end{abstract}

Keywords: nuclear medicine, radionuclide calibrator, activity. 


\section{INTRODUÇÃO}

Medicina Nuclear é uma especialidade da medicina que visa, principalmente, o diagnóstico de doenças. Apresenta vantagens como a de permitir a detecção precoce de doenças que não são diagnosticadas em outros exames, assim fazendo com que a chance de um prognóstico bem sucedido seja maior. A quantidade de material radioativo introduzido é controlada, de forma que o exame seja eficiente, mas que apresente riscos mínimos ao paciente relativos ao desenvolvimento de um câncer tardio. Dentre os principais radionuclídeos utilizados na medicina nuclear, estão o ${ }^{99 \mathrm{~m}} \mathrm{Tc}$, utilizado em cerca de $90 \%$ dos exames de diagnóstico, e o ${ }^{131} \mathrm{I}$, comumente aplicado nas investigações da tireóide ou para tratamento de câncer e hipertireoidismo.

A medida da atividade de uma solução radioativa a ser administrada é obtida por meio do uso do medidor de atividade, denominado ativímetro. Para que a atividade a ser administrada não seja nem maior e nem menor que o valor adequado, é fundamental que as calibrações sejam rastreadas aos padrões dos laboratórios nacionais de metrologia, de modo a não expor os pacientes a doses desnecessárias. A exatidão necessária para a medida de atividade de diferentes radionuclídeos é especificada por organismos com responsabilidades legais nesta área, por meio de normas ou recomendações. No Brasil, a exatidão requerida está expressa no Regulamento CNEN NE- 3.05 da Comissão Nacional de Energia Nuclear (CNEN, 2013), admitindo desvios de até $\pm 10 \%$, com um nível de confiança de $90 \%$.

Os ativímetro são calibrados pelos fabricantes com padrões em geometrias definidas e qualquer alteração nestas especificações pode gerar uma leitura significativamente diferente da atividade correta. Deve-se considerar que os valores desses fatores de correção dependem da energia dos fótons emitidos pelos radionuclídeos. Cada exame realizado necessita de um determinado volume e atividade de radiofármaco. Além disso, também é importante que se conheça os fatores de dependência de cada recipiente tais como: o volume do líquido e a geometria do suporte (Correia, 2012). Outros fatores também acarretam em incertezas na leitura da atividade em um ativímetro, como são mostrados na Tabela 1. A incerteza do tipo A é estimada a partir de uma série de observações repetidas, baseando-se na distribuição estatísticas dos resultados. 
Os estimadores das incertezas tipo A são definidos pelo desvio padrão experimental e pelo desvio padrão experimental da média. A incerteza do tipo B é obtida por julgamento científico ou por meio de informações sob a variação dos parâmetros de medição.

A incerteza padrão combinada caracteriza a dispersão dos valores medidos. Para o cálculo, utiliza-se a regra de combinação de variâncias partindo-se das incertezas padrões de combinação de cada componente do tipo A e do tipo B. A incerteza padrão combinada é muito usada para expressar a incerteza de um resultado de medição, mas muitas vezes é necessário definir um intervalo no qual o valor verdadeiro se situa. Este intervalo é denominado intervalo de confiança e a incerteza, de incerteza expandida (U), a qual é calculada multiplicando-se a incerteza padrão combinada por um fator de abrangência k (INMETRO, 2007).

Tabela 1: Fontes de incerteza na leitura da atividade em um ativímetro.

\section{Descrição do}

componente

\section{Como foi estimado}

Tipo de

incerteza

\begin{tabular}{lcc}
\hline Repetitividade & Desvio padrão de uma série de medidas & $\mathrm{A}$ \\
\hline Meia-vida & Incerteza devido à incerteza na meia-vida & $\mathrm{B}$ \\
\hline Impurezas & Incerteza nas atividades das impurezas detectadas & $\mathrm{B}$ \\
\hline Massa ou volume & Incerteza na massa ou volume da amostra a medir & $\mathrm{B}$ \\
\hline Resolução da escala & $\pm 0,5$ do último algarismo significativo da leitura da atividade & $\mathrm{B}$ \\
\hline $\begin{array}{l}\text { Correção para } \\
\text { geometria }\end{array}$ & Incerteza no fator de correção para a geometria & $\mathrm{B}$ \\
\hline
\end{tabular}

A fim de investigar o desempenho em qualidade das medições de rotina dos Serviços de Medicina Nuclear (SMN), desde 1998, o Laboratório Nacional de Metrologia das Radiações Ionizantes/Instituto de Radioproteção e Dosimetria (LNMRI/IRD) vem conduzindo um programa de intercomparação para medições atividade de radiofármacos administrados em pacientes de medicina nuclear. O programa é importante para garantir a confiabilidade dos resultados e promover o controle de qualidade, pois a literatura mostra que alguns desses equipamentos mal calibrados, ou com intervalos de tempo muito longos entre as calibrações, 
apresentam valores errôneos das medidas das atividades, podendo submeter os pacientes a doses de radiação desnecessárias ou insuficientes, prejudicando a qualidade da imagem (Tauhata et al, 2008).

Baseado no histórico de desempenho apresentados pelos SMN durante o período que participaram do programa de intercomparação do LNMRI/IRD, pode ser determinado fatores de correção para a atividade, a fim de determinar com melhor exatidão a atividade real a ser administrada. Com os valores de atividade corrigida obtidos, pode-se empregar estes valores para calcular com maior exatidão a dose efetiva recebida pelo paciente, bem como estimar o risco de indução de câncer (ICRP, 2007).

\section{MATERIAIS E MÉTODOS}

\subsection{DETERMINAÇÃO DO FATOR DE CORREÇÃO NA ATIVIDADE ADMINISTRADA}

A partir dos resultados obtidos na intercomparação de ativímetros, para os radionuclídeos ${ }^{99 m}$ Tc e ${ }^{131} \mathrm{I}$, em frascos padrão contendo, cada um, um volume de $5 \mathrm{ml}$ de solução radioativa, foram calculados os fatores de correção de atividade administrada aos pacientes de Medicina Nuclear. Os valores de referência das amostras foram obtidos empregando o sistema de medição padrão e rastreado a câmara de ionização padrão secundário modelo CENTRONIC IG11 do LNMRI.

O desempenho dos ativímetros dos SMN é avaliado em função da razão R, obtida pelo quociente do valor médio $\bar{X}$ das medidas realizadas no ativímetro do SMN, pelo valor de referência $X_{\mathrm{R} \text {, }}$ determinado no ativímetro do LNMRI, calculada segundo a equação (1):

$$
R=\frac{\bar{X}}{X_{R}}
$$

Se o parâmetro R apresentar valores entre 0.9 e 1.1, isto é, um intervalo com desvio relativo de $\pm 10 \%$, o desempenho é considerado em conformidade com os requisitos estabelecidos pela CNEN. Quanto mais próximo de 1 for o valor de R, melhor o desempenho alcançado pelo SMN. 
A avaliação final do desempenho do SMN participante é feita utilizando um critério diferente do parâmetro R, mas ainda dentro dos limites de $\pm 10 \%$ : a Equivalência Metrológica, expressa por dois parâmetros: o desvio do $\operatorname{SMN}\left(\mathrm{D}_{\mathrm{i}}\right)$ em relação ao valor de referência $\left(\mathrm{X}_{\mathrm{R}}\right)$ do LNMRI, e a incerteza combinada $\left(\mathrm{U}_{\mathrm{i}}\right)$ correspondente, com nível de confiança de aproximadamente $95 \%$, ou seja, $\mathrm{k}=2$. O desvio $\mathrm{D}_{\mathrm{i}}$ é obtido por:

$$
D_{i}=\left(X_{i}-X_{R}\right)
$$

Para os resultados das sucessivas comparações (i) de um radionuclídeo, o desvio do SMN ( $\left.\mathrm{D}_{\mathrm{i}}\right)$ é expresso por:

$$
\bar{d}=\frac{1}{n} \sum_{1}^{n}\left(\frac{D_{i}}{X_{R i}}\right)
$$

Conhecido o valor do desvio médio $\bar{d}$, pode-se corrigir o valor da atividade administrada $\left(\mathrm{A}_{\mathrm{j}}\right)$, obtido do registro de um determinado procedimento no prontuário do SMN, buscando obter uma estimativa do valor da atividade real administrada, ou seja:

$$
\left(A_{j}\right)_{\text {real }}=(1+\bar{d}) A_{j}
$$

O fator de correção da atividade está relacionado com o desvio médio $\bar{d}$ obtido pelo SMN a partir da equação:

$$
f=(1+\bar{d})
$$


O valor da incerteza combinada é calculado de acordo com a equação 6, assumindo $k=2$ (RATEL, 2005), onde $u_{i}$ e $u_{j}$ são respectivamente as incertezas de $X_{i}$ e $X_{R}$.

$$
U_{D_{i}}=k\left[\left(1-\frac{2}{n}\right) u_{i}^{2}+\left(\frac{1}{n^{2}}\right) \sum_{i}^{n} u_{j}^{2}\right]^{\frac{1}{2}}
$$

\section{RESULTADOS E DISCUSSÕES}

Os valores obtidos para o fator de correção da atividade administrada, para cada SMN, são apresentados na Tabela 2. Os fatores de correção obtidos são as médias dos resultados das comparações que cada hospital participou ao longo dos anos para o ${ }^{131} \mathrm{I}$ e ${ }^{99 \mathrm{~m}} \mathrm{Tc}$ e calculados a partir da equação (5).

Tabela 2: Fatores de correção de atividade.

\begin{tabular}{|c|c|c|}
\hline \multirow{2}{*}{ SMN } & \multicolumn{2}{|c|}{ Fator de Correção de Atividade } \\
\hline & ${ }^{99 m} \mathbf{T C}$ & ${ }^{131} \mathbf{I}$ \\
\hline POA1 & $1,0008 \pm 0,0161$ & $1,0172 \pm 0,0339$ \\
\hline POA2 & $0,9974 \pm 0,0325$ & $1,1531 \pm 0,2768$ \\
\hline RIO & $1,1266 \pm 0,0101$ & $1,2914 \pm 0,0135$ \\
\hline
\end{tabular}

Pode ser observado que apenas o SMN POA1 apresentou um valor dentro dos limites de aceitação de $\pm 10 \%$, para ambos os radioisótopos. O SMN POA2 apresentou um valor de leitura da atividade de, aproximadamente, $15 \%$ acima do valor de referência para o ${ }^{131}$ I. Já o SMN RIO apresentou valores de leitura de atividade acima do valor de referência para ambos os radioisótopos, aproximadamente $13 \%$ para o ${ }^{99 m}$ Tc e $30 \%$ para o ${ }^{131} \mathrm{I}$. Para o caso da leitura de atividade de ${ }^{131}$ I no SMN RIO, o resultado obtido é mais preocupante devido ao uso deste radioisótopo também para terapia. Consequentemente, o paciente de terapia recebe uma 
atividade muito menor que a prescrita, podendo comprometer no tratamento e aumentando consideravelmente o risco de falha no tratamento.

Neste trabalho foram avaliados 1496 pacientes adultos de diagnóstico em dois SMN de Porto Alegre e um SMN do Rio de Janeiro. Os exames onde a diferença entre a atividade administrada e a atividade administrada corrigida em pacientes adultos é mais significativa estão apresentados nas figuras 1 a 4 .

Figura 1: Valores médios de atividade e atividade corrigida obtidos no exame de controle renal qualitativo com ${ }^{99 m}$ Tc DMSA.

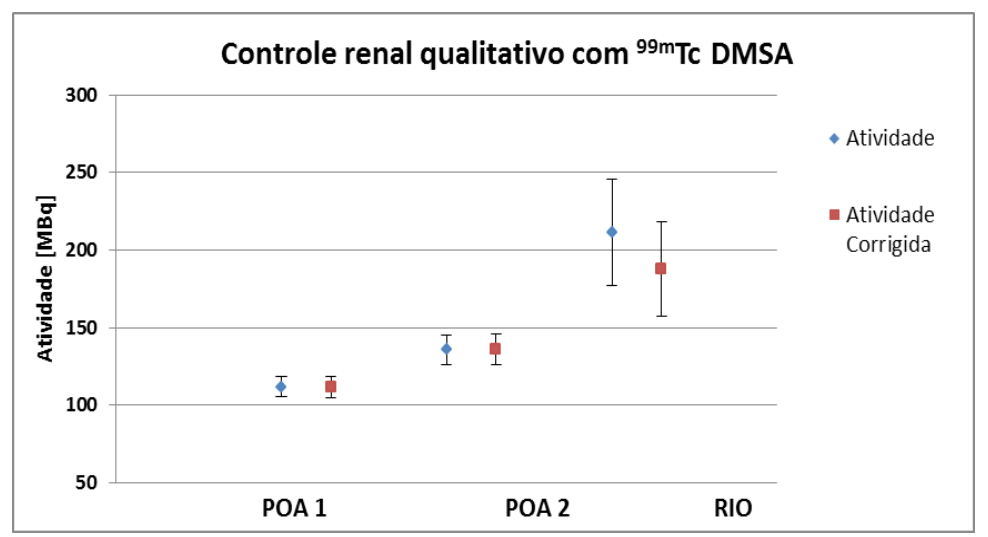

Figura 2: Valores médios de atividade e atividade corrigida obtidos no exame de estudo renal dinâmico com ${ }^{99 m}$ Tc DTPA em pacientes adulto.

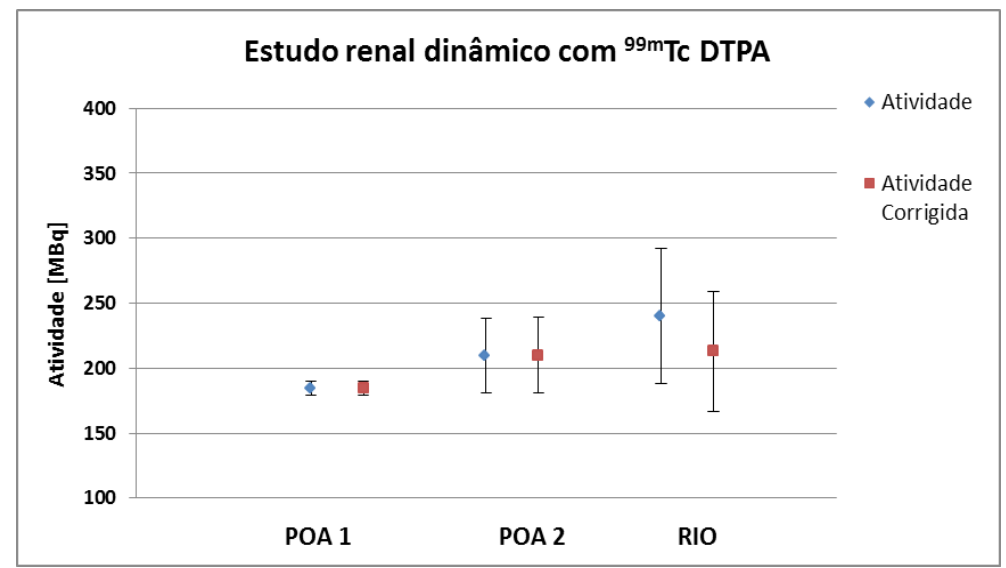


Figura 3. Valores médios de atividade e atividade corrigida obtidos no exame de cintilografia óssea com ${ }^{99 m}$ TcMDP em pacientes adultos

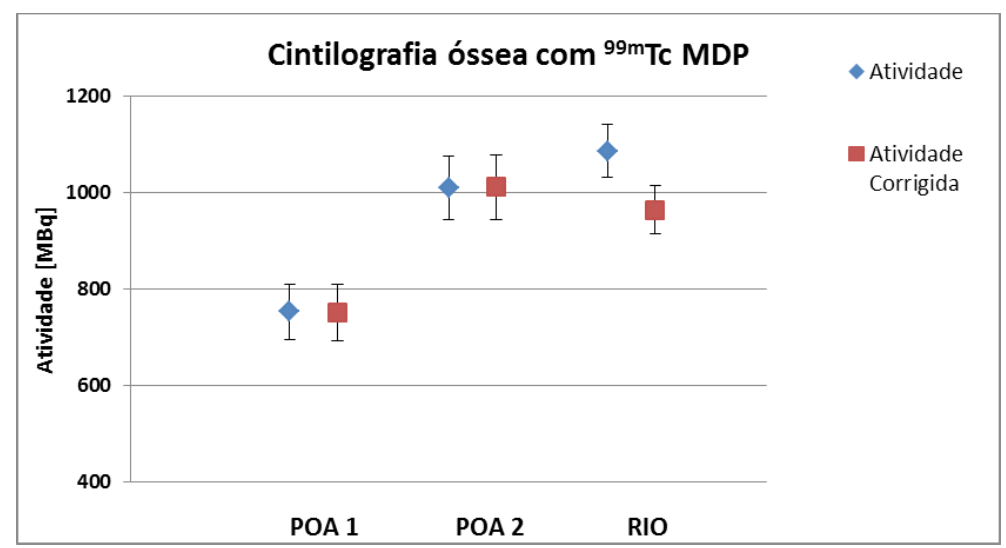

Figura 4: Valores médios de atividade e atividade corrigida obtidos no exame de cintilografia da tireóide com ${ }^{131}$ I em pacientes adultos.

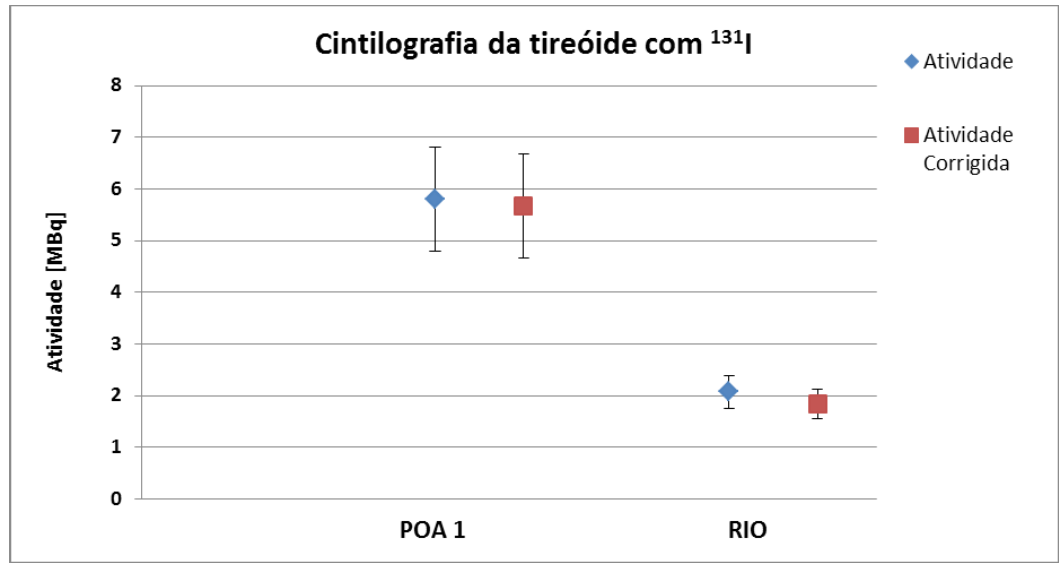

Como pode ser observado nas figuras anteriores, os valores médios de atividade administrada nos pacientes variam de acordo com o SMN. Apesar desta diferença, todos os valores de atividade administrada estão de acordo com as recomendações internacionais (ACR, 2013; SNM, 2006; SNM 2003). Porém, observa-se uma considerável variação entre a atividade administrada e a atividade corrigida para os exames realizados no SMN RIO. Esta variação é maior no exame 
de cintilografia da tireóide, onde a atividade administrada no paciente recebida pelo paciente é menor em aproximadamente $23 \%$.

Neste trabalho, foram avaliados pacientes pediátricos apenas para exames envolvendo ${ }^{99 \mathrm{~m}} \mathrm{Tc}$, pois exames de diagnóstico com ${ }^{131}$ I não apresentaram um número de pacientes estatisticamente significativo. A figura 5 mostra os resultados para o SMN RIO, nos exames pediátricos estudo renal dinâmico com ${ }^{99 m}$ Tc DTPA e controle renal qualitativo com ${ }^{99 m}$ Tc DMSA.

Figura 5: Valores médios de atividade e atividade corrigida obtidos em exames de investigação renal para pacientes pediátricos no SMN RIO.

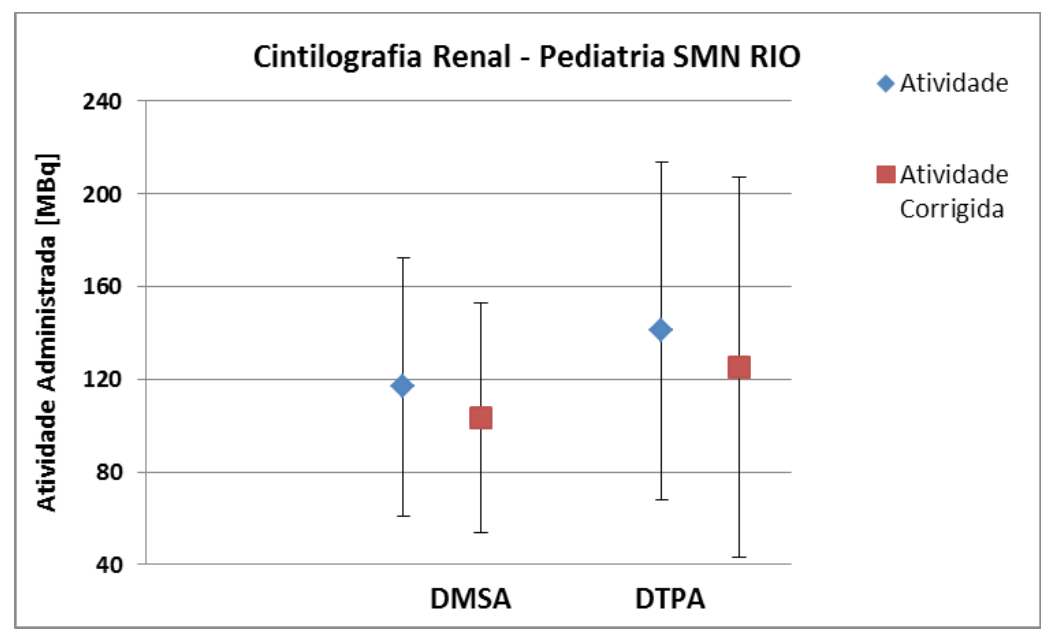

Observa-se uma diferença considerável entre a atividade administrada e a atividade corrigida. As consequências desta diferença não são muito críticas, pois a atividade corrigida foi inferior a atividade lida no ativímetro antes da administração, causando assim uma dose efetiva inferior no pacientes pediátrico. Porém, essa redução na atividade pode acarretar em problemas na obtenção da imagem e, consequentemente, falhas no diagnóstico. As atividades administradas em pacientes pediátricos exigem uma atenção maior, pois, mesmo sendo as atividades inferiores àquelas administradas em adultos, a dose recebida por esses pacientes é maior. Isso se deve ao fato que o organismo de crianças ainda está em desenvolvimento e suas células se dividem mais rapidamente, consequentemente a radiossensibilidade é maior. Os riscos relativos de certos efeitos negativos após uma exposição à radiação durante os primeiros dez anos de vida são de 3 a 
4 vezes maiores em comparação com uma exposição entre 30 a 40 anos de idade e de 5 a 7 vezes maiores em comparação com uma exposição após a idade de 50 anos (IAEA, 2001).

\section{CONCLUSÃO}

Para a proteção radiológica de pacientes em medicina nuclear, é importante que a atividade administrada seja a mais exata possível dentro dos limites de exatidão recomendados pela CNEN. Para as medições realizadas com ${ }^{99 \mathrm{~m}} \mathrm{Tc}$ e ${ }^{131} \mathrm{I}$, Porto Alegre apresenta melhor desempenho em comparação ao Rio de Janeiro. Em relação ao SMN RIO, o desempenho para o ${ }^{131}$ I é preocupante, pois uma diferença de $30 \%$ na atividade administrada no paciente, principalmente em terapia, pode prejudicar o tratamento, aumentando o risco de falha no tratamento.

A diferença entre a atividade reportada pelo hospital e a atividade de referência obtida pelo LNMRI é devida a medições incorretas nos SMN. Não temos condições e nem foi de interesse deste trabalho verificar se o ativímetro estava descalibrado ou se testes de controle de qualidade foram feitos. Apenas os valores de atividade administrada registrados nos prontuários médicos foram levados em consideração. Este trabalho não visa avaliar como os hospitais usam os seus ativímetros, se corretamente ou incorretamente, mas apenas mostrar que medições incorretas, se corrigidas implicam em redução de riscos.

No Brasil os fabricantes de ativímetros, todos estrangeiros, não mantem escritórios ou laboratórios que oferecem serviços de manutenção ou assistência técnica desses equipamentos caso eles apresentem problemas e necessitem de um ajuste como, por exemplo, uma nova calibração.

O único meio de verificar se o usuário está medindo corretamente a atividade de um determinado radiofármaco, numa geometria específica, é comparar sua medida com uma fonte padrão certificada (na mesma geometria) fornecida pelo LNMRI, que é o laboratório designado pelo INMETRO para atuar na área de metrologia das radiações ionizantes no Brasil.

Desta maneira, o desvio entre o valor medido pelo usuário e o valor certificado fornecido pelo LNMRI (que chamamos de fator de correção) pode ser usado para corrigir a leitura deste ativímetro obtendo a atividade correta, dentro das incertezas estabelecidas por essas medições.

Portanto, não estamos usando a comparação para calibração do equipamento, mas apenas um meio para que o usuário possa corrigir a leitura da atividade para obter um valor correto devido a 
essa deficiência de assistência técnica dos fabricantes dos ativímetros em operação nos serviços de medicina nuclear brasileiros. Atividades corretamente medidas administradas em pacientes terapêuticos são extremamente importantes no cálculo de doses.

\section{REFERÊNCIAS}

ACR - American College Of Radiology. ACR-SPR Practice Guideline for the Performance of Renal Scintigraphy. Res. 51, 2013.

BALON, H. R. et al. Society of Nuclear Medicine Procedure Guideline for Thyroid Scintigraphy. 12: 1-4, 2006.

CNEN-NN-3.05. COMISSÃO NACIONAL DE ENERGIA NUCLEAR. Requisitos de radioproteção e segurança para serviços de medicina nuclear. CNEN, 2013.

CORREIA, A. R.; IWAHARA, A.; TAUHATA, L.; REZENDE, E. A.; CHAVES, T. O. ; DE OLIVEIRA, A.E. ; DE OLIVEIRA, E.M. Fatores de correção volumétrica na medição de atividade de Tc-99m e I-123 em calibradores de radionuclídeos. RB. Radiologia Brasileira (Impresso), v. 45, p. 93-97, 2012.

DONOHOE K. J. et al. Society of Nuclear Medicine Procedure Guideline for Bone Scintigraphy. Nucl. Med. Technol., 30(12): B99-B103, 2003.

ICRP - International Commission on Radiological Protection. The 2007 Recommendations of the International Commission on radiological Protection. ICRP Publication 103. Oxford, Pergamon Press, 2007.

INMETRO. VIM - Vocabulário Internacional de Termos Fundamentais e Gerais de Metrologia. Instituto Nacional de Metrologia e Normalização e Qualidade Industrial, INMETRO. 5 5 Ed. Rio de Janeiro, SENAI, 2007.

IAEA - International Atomic Energy Agency. Radiological Protection of Patients in Diagnostic and Interventional Radiology, Nuclear Medicine and Radiotherapy. IAEA Publication 1113, 2001. 
RATEL, G. Evaluation of the uncertainty of the degree of equivalence. Metrologia 42, 140-144, pp. 141, 2005.

TAUHATA , L.; IWAHARA, A.; OLIVEIRA, A. E.; YACOVENCO , M.; REZENDE, E. A.; DELGADO, J. U.; SILVA, C. J. DA; SANTOS, J. A.; ALABARSE, F.; XAVIER,A.M. Proficiency tests in the determination of activity of radionuclides in radiopharmaceutical products measured by nuclear medicine services in 8 year of comparison programs in Brazil. Appl. Rad. Isotopes V.66, p.981 - 987, 2008. 